\title{
BASIS OF THE PROPERTIES OF WEIGHTED EXPONENTIAL SYSTEMS WITH EXCESS
}

\begin{abstract}
The main aim of this paper is the determination of a class of such functions for which a weighted exponential system becomes complete and minimal in appropriate space when exactly one of its terms is eliminated. It is shown that the system, obtained in this way cannot be a Schouder basis in this space. The last fact shows that Muckenhoupt-type criterion for the exponential system to be the Schauder basis in Lebesgue spaces after elimination of an element does not exist. This paper generalizes the results of the paper by E.S. Golubeva.
\end{abstract}

Key words: system of weighted exponentials, Muckenhoupt condition.

\section{Introduction}

The basis properties (completeness, minimality and Schauder basicity) of systems of the form $\left\{\omega(t) \varphi_{n}(t)\right\}$, where $\left\{\varphi_{n}(t)\right\}$ is an exponential or trigonometric (cosine or sine) systems have been investigated in several papers (see, for example, [1-16]). To our knowledge, first result in this direction is [1] in which Babenko gave an example $\left\{|t|^{\alpha} \cdot e^{i n t}\right\}_{n \in Z}$, where $|\alpha|<\frac{1}{2}$ and $\alpha \neq 0$, answering in the affirmative a question of Bari ([17]) on the existence of normalized basis for $L_{2}(-\pi, \pi)$ that is not a Riesz basis. The result of Babenko ([1]) was then extended by V.F.Gaposhkin in his famous paper [14], where, in particular, some sufficient condition (on the weight function $\omega(t)$ ) for the system $\left\{\omega(t) \cdot e^{i n t}\right\}_{n \in Z}$ to be a basis in $L_{2}(-\pi, \pi)$ was found. And eventually, necessary and sufficient condition on the weight function $\omega(t)$ whcih ensures the Schauder basicity of the exponential system $\left\{e^{i n t}\right\}_{n \in Z}$ in weighted Lebesgue space $L_{p, \omega()}(-\pi, \pi)$ has been obtained (see, for example [16]); such a condition is the Muckenhaupt condition with respect to the weight function $\omega(t)$ :

$$
\sup _{I}\left(\frac{1}{|I|} \int_{I} \omega(t) d t\right)\left(\frac{1}{|I|} \int_{I} \omega^{-\frac{1}{p-1}}(t) d t\right)^{p-1}<\infty
$$

where sup is taken over all intervals $I$ and $|I|$ is the length of the interval $I$. Note that study of basicity properties of a system in weighted Lebesgue spaces $L_{p, \omega()}$ is equivalent to the study of analogous properties of this system with corresponding degenerate coefficient in the "ordinary"Lebesgue space $L_{p}$. Therefore, the mentioned criterion can also be considered as a necessary and sufficient condition for the Schauder basicity in $L_{p}$ of the exponential system with degenerate coefficients $\left\{\omega(t) e^{i n t}\right\}_{n \in Z}$.

There are concrete examples of weight function $\omega(t)$ for which the system $\left\{\omega(t) e^{i n t}\right\}_{n \in Z}$ itself is not complete and minimal but becomes so when some of its terms are eliminated. For example, it is proved in [8] that, a system $\left\{t e^{i n t}\right\}_{n \in Z}$ is not complete and minimal (and hence Schauder basis) in $L_{2}(-\pi, \pi$ ) but becomes complete and minimal when one of its elements is eliminated from the original system; more precisely, a system $\left\{t e^{i n t}\right\}_{n \in Z /\{0\}}$ is complete and minimal system in $L_{2}(-\pi, \pi)$, but it is not a Schauder basis in it. It is also mentioned in [8] that the indicated statement about the system remains valid when any one of its terms is eliminated.

We generalize the indicated result of the paper [8] to the most general case of the system $\left\{\omega(t) e^{i n t}\right\}_{n \in Z}$, where $\omega(t)$ is any function.

The aim of this note is the determination of the class of all functions $\omega(t)$ for which the system $\left\{\omega(t) e^{i n t}\right\}_{Z}$ becomes complete and minimal in $L_{p}(-\pi, \pi), 1<p<\infty$, space when exactly one of its terms is eliminated. It is also shown that the system, obtained from the system $\left\{\omega(t) e^{i n t}\right\}_{Z}$ in this way (by elimination of an element) cannot be a Schauder basis in $L_{p}(-\pi, \pi)$ space.

\footnotetext{
${ }^{1}$ C Shukurov A.Sh., 2018

Shukurov Aydin Shukur (ashshukurov@gmail.com), Institute of Mathematics and Mechanics, NAS of Azerbaijan, 9, B. Vahabzade, Baku, Az1141, Azerbaijan.
} 


\section{Auxiliary facts}

We will use some auxiliary facts which are of some interest in their own too.

Lemma 1. If the system $\left\{\omega(t) e^{i n t}\right\}_{n \in Z / k_{0}}$ is a minimal system in $L_{p}(-\pi, \pi)$, then it has a biorthogonal system $\left\{b_{n}(t)\right\}_{Z / k_{0}}$ which is of the following form:

$$
b_{n}(t)=\frac{e^{i n t}+\xi_{n} e^{i k_{0} t}}{\omega(t)}
$$

where $\xi_{n}$ are some complex numbers.

Proof. The fact that $\left\{\omega(t) e^{i n t}\right\}_{n \in Z / k_{0}}$ has a biorthogonal system follows from its minimality. Denote the biorthogonal system by $\left\{b_{n}(t)\right\}_{Z / k_{0}}$. Take arbitrary natural number $n \neq k_{0}$. By the definition of the biorthogonal system

$$
\int_{-\pi}^{\pi} b_{n}(t) \omega(t) e^{i k t} d t=0, \forall k \neq n, k_{0}
$$

and

$$
\int_{-\pi}^{\pi} b_{n}(t) \omega(t) e^{i n t} d t=1 .
$$

The relations (2), along with the fact that the Fourier coefficients of a summable function with respect to an exponential system is unique, imply that there are some complex numbers $\alpha_{n}$ and $\xi_{n}$ such that

$$
b_{n}(t)=\frac{\alpha_{n} e^{i n t}+\xi_{n} e^{i k_{0} t}}{\omega(t)} .
$$

Substitutung it into (3) and taking into account that $\left\{e^{i n t}\right\}$ is an orthonormal system, we find that $\alpha_{n}=1$ for all $n$. This proves the relation (1). The Lemma is proved.

Let $\left\{\xi_{n}\right\}$ be any sequence of numbers and $k_{0}$ any integer. For simplicity, we will make use of the following denotation:

$$
\Phi_{n}(t)=e^{i n t}-\xi_{n} e^{i k_{0} t}
$$

Lemma 2. A function $\Phi_{n}(t)$, where $n \neq k_{0}$, may have only simple zeros.

Proof. Assume that there is a point $t_{0}$ for which

$$
\Phi_{n}\left(t_{0}\right)=0, \Phi_{n}^{\prime}\left(t_{0}\right)=0 .
$$

These equalities can be written in the following form:

$$
e^{i n t_{0}}-\xi_{n} e^{i k_{0} t_{0}}=0
$$

and

$$
n e^{i n t_{0}}-k_{0} \xi_{n} e^{i k_{0} t_{0}}=0
$$

accordingly. These equalities imply that $n=k_{0}$ which is impossible by the condition of the lemma.

The lemma is proved.

Lemma 3. Let $A \subset[-\pi, \pi]$ be such that $\Phi_{n}(t)=0$ for all $n \in Z /\left\{k_{0}\right\}, t \in A$. Then the set $A$ consists of at most two points and it is two-element set only if $A=\{-\pi, \pi\}$.

Proof. Let $t_{1}, t_{2} \in[-\pi, \pi]$ be such that

$$
e^{i n t_{1}}-\xi_{n} e^{i k_{0} t_{1}}=0
$$

and

$$
e^{i n t_{2}}-\xi_{n} e^{i k_{0} t_{2}}=0
$$

for all $n \neq k_{0}$. These two relations are possible if and only if

$$
\frac{\left(n-k_{0}\right)\left(t_{2}-t_{1}\right)}{2 \pi} \in Z
$$

for all $n \neq k_{0}$. Taking, in particular, $n=k_{0}+1$, we obtain from the last relation that $t_{2}-t_{1}$ is an integer multiply of $2 \pi$. But this is possible if and only if $t_{1}=-\pi, t_{2}=\pi$ or $t_{2}=-\pi, t_{1}=\pi$. Thus, the set $A$ may contain at most two points and it contains two elements only if $A=\{-\pi, \pi\}$.

The lemma is proved 
Lemma 4. Let $\xi$ be any complex number and $n, m$ any integers such that $n \neq m$. Then the function $e^{i n t}-\xi \cdot e^{i m t}$ may have only finite number of zeroes in the segment $[-\pi, \pi]$.

Proof. Assume the contrary: the function $e^{i n t}-\xi \cdot e^{i m t}$ has an infinite number of zeroes. Let $\left\{z_{n}\right\}_{n=1}^{\infty} \subset$ $\subset[-\pi, \pi]$ be its zeroes. By the Bolzano-Weierstrass theorem, the sequence $\left\{z_{n}\right\}_{n=1}^{\infty}$ has a limit point in $[-\pi, \pi]$. Therefore, since the function $e^{i n z}-\xi \cdot e^{i m z}$ is an entire function on the whole complex plane, the uniqueness theorem for analytic functions implies that $e^{i n t}-\xi \cdot e^{i m t} \equiv 0$ on the segment $[-\pi, \pi]$. This means that the system of functions $\left\{e^{i n z}, e^{i m z}\right\}$ is linearly independent system. Contradiction: since it is orthonormal, it cannot be linearly independent. The Lemma is proved.

\section{Main result and its proof}

The aim of this paper is to prove the following

Main Theorem. Let $k_{0}$ be any integer. The system $\left\{\omega(t) e^{i n t}\right\}_{n \in Z /\left\{k_{0}\right\}}$ is complete and minimal in $L_{p}(-\pi, \pi), 1<p<\infty$, space if and only if $\omega(t) \in L_{p}(-\pi, \pi), \frac{1}{\omega(t)} \notin L_{q}(-\pi, \pi)$ and besides

1) there is a (unique) point $t_{0} \in[-\pi, \pi]$ such that $\frac{t-t_{0}}{\omega(t)} \in L_{q}(-\pi, \pi)$; or

2) $\frac{(t-\pi)(t+\pi)}{\omega(t)} \in L_{q}(-\pi, \pi)$.

Proof. Necessity. The validity of $\omega(t) \in L_{p}(-\pi, \pi)$ is evident.

Let the system $\left\{\omega(t) e^{i n t}\right\}_{n \in Z /\left\{k_{0}\right\}}$ be complete and minimal in $L_{p}(-\pi, \pi)$. Then

$$
\frac{1}{\omega(t)} \notin L_{q}(-\pi, \pi)
$$

Assume the contrary:

$$
\frac{1}{\omega(t)} \in L_{q}(-\pi, \pi)
$$

Then $\frac{1}{\omega(t)} e^{i k_{0} t} \in L_{q}(-\pi, \pi)$ and besides this, it is evident that the function $\frac{1}{\omega(t)} e^{i k_{0} t}$ is not trivial (is not equivalent to zero) and

$$
\int_{-\pi}^{\pi} \omega(t) e^{i n t} \frac{1}{\omega(t)} e^{i k_{0} t} d t=0
$$

for all $n \in Z, n \neq k_{0}$. These observations show that the system $\left\{\omega(t) e^{i n t}\right\}_{n \in Z /\left\{k_{0}\right\}}$ is not complete. Thus, our assumption is false - (4) is valid.

Since the system $\left\{\omega(t) e^{i n t}\right\}_{n \in Z /\left\{k_{0}\right\}}$ is minimal, by Lemma 1 it has a biorthogonal that is of the following form:

$$
b_{n}(t)=\frac{e^{i n t}-\xi_{n} e^{i k_{0} t}}{\omega(t)}, \forall n \neq k_{0} .
$$

If the function $e^{i n t}-\xi_{n} e^{i k_{0} t}$ has no zeros on the segment $[-\pi, \pi]$ for some index $n$, then the representation (5), the fact that $b_{n}(t) \in L_{q}(-\pi, \pi)$ and the continuity of the function $e^{i n t}-\xi_{n} e^{i k_{0} t}$ imply that $\frac{1}{\omega(t)} \in$ $\in L_{q}(-\pi, \pi)$ which contradicts to $(4)$.

Let $n_{0}$ be any natural number satisfying the condition $n_{0} \neq k_{0}$. By Lemma 4 , the function $e^{i n_{0} t}-\xi_{n} e^{i k_{0} t}$ has finite number of zeros $z_{1}, \ldots, z_{m}$ on the segment $[-\pi, \pi]$. With this in mind, using the representation (5) of the biorthogonal system, the fact that $b_{n}(t) \in L_{q}(-\pi, \pi)$ for all $n \neq k_{0}$ and the condition $\frac{1}{\omega(t)} \notin L_{q}(-\pi, \pi)$, we obtain that there is a nonempty subset $A \subset[-\pi, \pi]$ (consisting of the points $z_{1}, \ldots, z_{m}$ ) such that

$$
e^{i n t}-\xi_{n} e^{i k_{0} t}=0
$$

for all $n \in Z /\left\{k_{0}\right\}$. By Lemma 3, the set $A$ consists of at most two points and it contains two elements only if $A=\{-\pi, \pi\}$.

We treat the single point and two-point cases separately.

First, consider the case $A=\left\{t_{0}\right\}$, where $t_{0}$ is some number in $\left\{z_{1}, \ldots, z_{m}\right\}$. Write the function $b_{n_{0}}(t)$ in the following form

and consider an auxiliary function

$$
b_{n_{0}}(t)=\frac{t-t_{0}}{\omega(t)} \cdot \frac{e^{i n_{0} t}-\xi_{n} e^{i k_{0} t}}{t-t_{0}}
$$




$$
\Phi(t)= \begin{cases}\frac{e^{i n_{0} t}-\xi_{n_{0}} e^{i k_{0} t}}{t-t_{0}}, & \text { if } t \neq t_{0} \\ \Phi_{n_{0}}^{\prime}\left(t_{0}\right), & \text { if } t=t_{0} .\end{cases}
$$

Then, using the definition of the set $A$, taking into account that $b_{n}(t) \in L_{q}(-\pi, \pi)$ for all $n \neq k_{0}$ and using Lemma 2, it can be derived from here that

$$
\frac{t-t_{0}}{\omega(t)} \in L_{q}(-\pi, \pi)
$$

Now, consider the case $A=\{-\pi, \pi\}$. Write the function $b_{n_{0}}(t)$ in the following form

and consider a function

$$
b_{n_{0}}(t)=\frac{(t-\pi)(t+\pi)}{\omega(t)} \cdot \frac{e^{i n_{0} t}-\xi_{n_{0}} e^{i k_{0} t}}{(t-\pi)(t+\pi)}, \forall n \neq k_{0}
$$

$$
\Phi(t)= \begin{cases}\frac{e^{i n_{0} t}-\xi_{n_{0}} e^{i k_{0} t}}{(t-\pi)(t+\pi)}, & \text { if } t \in(-\pi, \pi) \\ -\frac{\Phi_{n_{0}}^{\prime}(\pi)}{2 \pi}, & \text { if } t=-\pi ; \\ \frac{\Phi_{n_{0}}^{\prime}(\pi)}{2 \pi}, & \text { if } t=\pi .\end{cases}
$$

Then, again, using the definition of the set $A$, taking into account that $b_{n}(t) \in L_{q}(-\pi, \pi)$ for all $n \neq k_{0}$ and applying Lemma 2, it can be shown that

$$
\frac{(t-\pi)(t+\pi)}{\omega(t)} \in L_{q}(-\pi, \pi)
$$

Sufficiency. First, consider the case 1): there is a unique point $t_{0} \in[-\pi, \pi]$ such that $\frac{t-t_{0}}{\omega(t)} \in L_{q}(-\pi, \pi)$. Let $k_{0}$ be any integer. If a function $f(t)$ is orthogonal to the system $\left\{\omega(t) \cdot e^{i n t}\right\}_{n \in Z /\left\{k_{0}\right\}}$,

$$
\int_{-\pi}^{\pi} f(t) \omega(t) e^{i n t} d t=0, \forall n \neq k_{0}
$$

then $f(t)=\frac{c \cdot e^{i k_{0} t}}{\omega(t)}$ for some constant $c$ (it is a consequence of the fact that Fourier coefficients of a summable function with respect to an exponential system is unique). Since $\frac{1}{\omega(t)} \notin L_{q}(-\pi, \pi), f(t)=\frac{c \cdot e^{i k_{0} t}}{\omega(t)} \in L_{q}(-\pi, \pi)$ if and only if $c=0$, i.e. $f(t) \equiv 0$. Thus, the relations (6 ) imply $f(t) \equiv 0$. This means that the system $\left\{\omega(t) e^{i n t}\right\}_{n \in Z /\left\{k_{0}\right\}}$ is complete in $L_{p}(-\pi, \pi)$.

Consider a function $b_{n}(t)$ defined by $(5)$, where $\xi_{n}=\frac{e^{i n t_{0}}}{e^{i k_{0} t_{0}}}$. Writing the function $b_{n}(t)$ in the form

$$
b_{n}(t)=\frac{t-t_{0}}{\omega(t)} \cdot \frac{e^{i n t}-\xi_{n} e^{i k_{0} t}}{t-t_{0}},
$$

taking into accout the relation $\frac{t-t_{0}}{\omega(t)} \in L_{q}(-\pi, \pi)$ and the fact that $t_{0}$ is a root of the function $e^{i n t}-\xi_{n} e^{i k_{0} t}$, we obtain that $b_{n}(t) \in L_{q}(-\pi, \pi)$ for all $n \in Z, n \neq k_{0}$; besides it, it is easy to see that

$$
\int_{-\pi}^{\pi} b_{n}(t) \omega(t) e^{i m t} d t=\delta_{n m}
$$

for all $n, m \neq k_{0}$, where $\delta_{n m}$ is a Kronecker symbol. Therefore, the system $\left\{b_{n}(t)\right\}$ is biorthogonal to $\left\{\omega(t) e^{i n t}\right\}_{n \in Z /\left\{k_{0}\right\}}$ and hence, the system $\left\{\omega(t) e^{i n t}\right\}_{n \in Z /\left\{k_{0}\right\}}$ is minimal in $L_{p}(-\pi, \pi)$ space.

The case 2) is treated similarly.

The theorem is proved.

This theorem immediately implies the following

Corollary. If the system $\left\{\omega(t) e^{i n t}\right\}_{Z}$ becomes complete and minimal in $L_{p}(-\pi, \pi)$ space when one of its terms is eliminated, then it also becomes complete and minimal in $L_{p}(-\pi, \pi)$ when any one of its terms is eliminated.

\section{Schauder basicity}

The result of the previous section characterizes the class of all functions $\omega(t)$ for which the system $\left\{\omega(t) e^{i n t}\right\}_{Z}$ becomes complete and minimal in $L_{p}(-\pi, \pi), 1<p<\infty$, space when exactly one (actually, by Corollary of the previous section, any one) of its terms is eliminated. Therefore it is natural to ask for 
condition on $\omega(t)$ which ensures Schauder basicity in $L_{p}(-\pi, \pi)$ of the system, obtained from the system $\left\{\omega(t) e^{i n t}\right\}_{Z}$ by elimination of exactly one of its terms. It turns out that such a condition does not exist:

Theorem. Let $\omega(t)$ be any function and $k_{0}$ be any integer. Then the system $\left\{\omega(t) e^{\text {int }}\right\}_{Z /\left\{k_{0}\right\}}$ is not a Schauder basis in $L_{p}(-\pi, \pi), 1<p<\infty$, space.

Proof. Assume the contrary: the system $\left\{\omega(t) e^{i n t}\right\}_{Z /\left\{k_{0}\right\}}$ is a Schauder basis in $L_{p}(-\pi, \pi)$. Then the function $\omega(t) e^{i k_{0} t}$ has an expansion (in $L_{p}$ norm) of the form

$$
\omega(t) e^{i k_{0} t}=\sum_{n \neq k_{0}}^{\infty} c_{n} \omega(t) e^{i n t} .
$$

Take arbitrary natural number $n \neq k_{0}$. Applying the biorthogonal system (5) to both sides of (7), we obtain that

Thus, the series

$$
c_{n}=\xi_{n}, \forall n \neq k_{0}
$$

$$
\sum_{n \neq k_{o}}^{\infty} \xi_{n} \omega(t) e^{i n t}
$$

is convergent. Therefore, by the necessary condition for the convergence of the series, $\lim _{n \rightarrow \infty}\left\|\xi_{n} \omega(t) e^{i n t}\right\|_{L_{p}}=$ $=0$. This equality and the identity $\left\|\xi_{n} \omega(t) e^{i n t}\right\|_{L_{p}}=\left|\xi_{n}\right| \cdot\|\omega(t)\|_{L_{p}}$ imply that $\lim _{n \rightarrow \infty} \xi_{n}=0$. But, on the other hand, as a consequence of the basicity, the system $\left\{\omega(t) e^{i n t}\right\}_{Z /\left\{k_{0}\right\}}$ is complete and minimal in $L_{p}(-\pi, \pi)$ and a closer look at the proof of Main Theorem from the previous section shows that the number $\xi_{n}$ in the definition of the biorthogonal system (5) satisfies the equality $\xi_{n}=\frac{e^{i n t_{0}}}{e^{i k_{0} t_{0}}}$ for all $n \in Z, n \neq k_{0}$, for some $t_{0} \in[-\pi, \pi]$. This yields a contradiction. The Theorem is proved.

Remark. It should be noted that this fact also follows from the more general result obtained in [18-20].

Note that negative results on Schauder basicity of some systems of a certain form were also studied earlier in papers [21-25].

Acknowledgement. The author is grateful to Professor B.T.Bilalov for encouraging discussion. The author also thanks N.J. Guliyev for his useful assistance.

\section{References}

[1] K.I. Babenko On conjugate functions. Doklady Akad. Nauk SSSR (N.S.) 62, (1948), pp. $157-160$ [in Russian].

[2] B.T. Bilalov, S.G. Veliyev On completeness of exponent system with complex coefficients in weight spaces. Trans. of NAS of Azer., 2005, Vol. XXV, no. 7, pp. 9-14 [in English].

[3] B.T. Bilalov, S.G. Veliyev Bases of eigenfunctions of two discontinuous differential operators. Diff. Uravn., 2006, Vol. 42, no. 9, pp. 190-192 [in English].

[4] K.S. Kazaryan, P.I. Lizorkin Multipliers, bases and unconditional bases of the weighted spaces B and SB. Trudy Mat. Inst. Steklov, no. 187 (1989), pp. 98-115 [in English].

[5] S.S. Pukhov, А.M. SedletskiД Bases of exponentials, sines, and cosines in weighted spaces on a finite interval. Dokl. Akad. Nauk, 425 (2009), no. 4, pp. 452-455 [in English].

[6] E.I. Moiseev On the basis property of sine and cosine systems in a weighted space. Differ. Uravn., 34 (1998), no. 1, pp. 40-44 [in English].

[7] E.I. Moiseev The basis property of a system of eigenfunctions of a differential operator in a weighted space. Differ. Uravn., 35 (1999), no. 2, pp. 200-205 [in English].

[8] E.S. Golubeva The System of Weighted Exponentials with Power Weights. Vestnik SamGU. Estestvenno-Nauchnaya Seriia, 2011, no. 2(83), pp. 15-25 [in Russian].

[9] Z.V. Mamedova On Basis Properties of Degenerate Exponential System. Applied Mathematics, 2012, 3, pp. 1963-1966 [in English].

[10] S.R. Sadigova, Z.V. Mamedova Frames from Cosines with the Degenerate Coefficients. American Journal of Applied Mathematics and Statistics, 2013, Vol. 1, no. 3, pp. 36-40 [in English].

[11] B. Bilalov, F. Guliyeva On the Frame Properties of Degenerate System of Sines. Journal of Function Spaces and Applications, 2012, Art. ID 184186, pp. 1-12 [in English].

[12] B.T. Bilalov, Z.V. Mamedova On the frame properties of some degenerate trigonometric systems. Dokl. Nats. Akad. Nauk Azerb., 68 (2012), no. 5, pp. 14-18 [in English].

[13] B.T. Bilalov, F.A. Gulieva, A completeness criterion for a double power system with degenerate coefficients, Sibirsk. Mat. Zh. 54 (2013), no. 3, 536-543. 
[14] V.F. Gaposhkin A generalization of the theorem of M. Riesz on conjugate functions Mat. Sb. N.S., 46(88), 1958, pp. 359-372 [in English].

[15] B.T. Bilalov, S.R. Sadigova Frame properties of a part of exponential system with degenerate coefficients in Hardy classes Georgian Mathematical Journal (accepted, will be published in Vol.23(2017)) [in English].

[16] R.A. Hunt, W.S. Young A weighted norm inequality for Fourier series. Bull. Amer. Math. Soc., 80 (1974), 274, p. 277. [in English].

[17] N.K. Bari Biorthogonal systems and bases in Hilbert space. Moskov. Gos. Univ. Uchenye Zapiski. Matematika, 148(4), (1951), 69-107 [in Russian].

[18] K.S. Kazarian The multiplicative completion of basic sequences in $L_{p}, 1<p<\infty$ to bases in $L_{p}$. Dokl. Akad. Nauk Arm. SSR, Vol. 62, 1976, pp. 203-209 [in English].

[19] K.S. Kazarian On the multiplicative completion of some incomplete orthonormal systems to bases in $L_{p}, 1<p<\infty$. Analysis Math, 1978, 4, pp. 37-52 [in English].

[20] K.S. Kazarian Multiplicative completion of certain systems. Izv. Akad. Nauk Armyan. SSR Ser. Mat., 1978, 13(4) [in English].

[21] L.V. Kritskov On the basis property of the system of functions $\left\{e^{i \alpha n t} \sin (n t)\right\}$. Dokl. Akad. Nauk, 346 (1996), no. 3, pp. 297-298 [in Russian].

[22] A.Sh. Shukurov Necessary condition for Kostyuchenko type systems to be a basis in Lebesgue spaces, Colloq. Math. 127 (2012), no. 1, 105-109.

[23] A.Sh. Shukurov Addendum to "Necessary condition for Kostyuchenko type systems to be a basis in Lebesgue spaces" [MR2945779]. Colloq. Math., 137 (2014), no. 2, pp. 297-298 [in English].

[24] A.Sh. Shukurov The power system is never a basis in the space of continuous functions. Amer. Math. Monthly, 122 (2015), no. 2, p. 137 [in English].

[25] A.Sh. Shukurov Impossibility of power series expansion for continuous functions. Azerb. J. Math., 6 (2016), no. 1, pp. 122-125 [in English].

\section{A.Ш. Шукюров ${ }^{2}$}

\section{О БАЗИСНЫХ СВОЙСТВАХ ВЗВЕШЕННЫХ ЭКСПОНЕНЦИАЛЬНЫХ СИСТЕМ С ИЗБЫТКОМ}

\footnotetext{
Целью настоящей работы является обобщение результатов Э.С. Голубева на основе свойств взвешенных экспоненциальных систем, опубликованных ранее в этом журнале, в наиболее общем случае.
}

Ключевые слова: система взвешенных экспоненциалов, условие Muckenhoupt.

Статья поступила в редакцию 28/II/2018.

The article received $28 / I I / 2018$.

\footnotetext{
${ }^{2}$ Шукюров Айдън Шукюр огль (ashshukurov@gmail.com), Институт Математики и Механики, Национальная Академия Наук Азербайджана, Az1141, Азербайджан, Баку, Б. Вахабзаде, 9.
} 